\title{
Politique
}

Politique

\section{Vaclav Havel : le premier président postmoderne?}

\section{Yves Boisvert}

Numéro 21, hiver 1992

URI : https://id.erudit.org/iderudit/040713ar

DOI : https://doi.org/10.7202/040713ar

Aller au sommaire du numéro

Éditeur(s)

Société québécoise de science politique

ISSN

0711-608X (imprimé)

1918-6584 (numérique)

Découvrir la revue

Citer cet article

Boisvert, Y. (1992). Vaclav Havel : le premier président postmoderne? Politique, (21), 71-96. https://doi.org/10.7202/040713ar

\section{Résumé de l'article}

Dans cet article, l'auteur tente de cerner la pensée philosophico-politique du dramaturge tchèque et ex-dissident Vaclav Havel. À la lumière de sa critique à l'égard de la modernité et de l'européocentrisme, l'auteur s'interroge sur la portée de la réflexion de celui qui fut porté à la présidence de son pays suite à la révolution de velours, et se demande si elle peut s'inscrire dans le sillon postmoderniste. d'utilisation que vous pouvez consulter en ligne.

https://apropos.erudit.org/fr/usagers/politique-dutilisation/ 


\title{
VACLAV HAVEL : \\ LE PREMIER PRÉSIDENT \\ POSTMODERNE ?
}

\author{
Yves Boisvert \\ Université du Québec à Montréal
}

Dans cet article, l'auteur tente de cerner la pensée philosophicopolitique du dramaturge tchèque et ex-dissident Vaclav Havel. À la lumière de sa critique à l'égard de la modernité et de l'européocentrisme, l'auteur s'interroge sur la portée de la réflexion de celui qui fut porté à la présidence de son pays suite à la révolution de velours, et se demande si elle peut s'inscrire dans le sillon postmoderniste.

L'année 1989 restera longtemps gravée dans nos mémoires comme l'un des moments historiques marquants de cette fin de siecle. L'important raz-de-marée anticommuniste qui a balayé l'Europe de l'Est confirme la fin d'une époque et l'avènement d'une nouvelle ère. Outre l'effondrement du honteux mur de Berlin, l'arrivée au pouvoir en Tchécosiovaquie du dramaturge-dissident Vaclav Havel a été l'un des événements qui a retenu l'attention au cours de cette année charnière.

Si quelques Occidentaux connaissaient les talents de dramaturge de l'ex-président tchécoslovaque, le nom de Vaclav Havel demeurait, avant la "révolution de velours", quasi inconnu de l'opinion publique internationale. II n'en est plus de même aujourd'hui.

Malgré l'intérêt qu'a suscité le président de la république tchèque et slovaque, ses idées politiques restent une grande énigme. La réflexion de Havel est d'autant plus riche qu'elle est ouverte et qu'elle s'inspire aussi bien de Husserl et de Heidegger que d'Aristote tout en offrant de nombreuses affinités avec les courants de la pensée occidentale contemporaine : la théorie des nouveaux mouvements 
sociaux, la pensée postmoderne et le communautarisme. Cet éclectisme engendre néanmoins plusieurs paradoxes et contradictions. Comme toute réflexion, celle de Havel a ses forces et ses faiblesses.

Havel représente l' «autre» pensée, et c'est justement parce que cette pensée est différente qu'elle peut nous être utile. Selon ses propres mots, elle est le miroir ou la caricature de nos sociétés modernes. C'est dans cet esprit que nous considérons la philosophie sociale et politique de Vaclav Havel comme un riche apport a l'avancement de la pensée politique contemporaine.

Dans le présent article, nous présentons une analyse interprétative d'un fragment de la pensée de cet auteur. Nous limitons ainsi notre étude à l'analyse de quatre thèmes philosophico-politiques : la critique de l'utopie occidentale; celle de la modernité; la redéfinition du concept de liberté; la présentation de son schéma postdémocratique.

A travers l'étude de ces quatre thèmes, nous nous demanderons quelle a été l'influence de la pensée "havelienne" sur le débat contemporain qu'inspire le courant de la postmodernité. Nous chercherons à savoir si une telle pensée infirme ce courant ou si, au contraire, elle lui fournit de nouveaux éléments théoriques. Notre thèse est que, même si plusieurs éléments de la réflexion que poursuit Havel alimentent le discours sur la postmodernité, cet écrivain ne peut pas être considéré comme un penseur postmoderne en raison surtout de son obsession phénoménologique quasi religieuse.

Sans nous engager dans la controverse dont fait l'objet la postmodernité, nous entendons par "postmoderne" le mouvement de pensée hétérogène qui affirme l'impossibilité, à l'heure où nos sociétés occidentales contemporaines sont dominées par la technoscience et par les communications de masse, de continuer à penser et à organiser nos communautés en fonction des idéaux modernes, conçus il y a plus de deux siècles. 
Lyotard dit avec insistance :

II ne s'agit pas là d'un abandon du projet moderne, comme le dit Habermas a propos de la postmodernité, mais de sa wliquidation». Ce qui s'inscrit alors dans la conscience européenne sinon occidentale, avec cet anéantissement, c'est de façon irreparable le soupcon que l'histoire universelle ne conduit pas surement "vers le mieux", comme disait Kant, ou plutot que l'histoires n'a pas nécessairement une finalité universelle'.

Selon cet auteur, ce ne sont donc pas les tenants de la postmodernité qui sont responsables de l'échec de la modernité. Elle s'est liquidée elle-même à travers différents événements qui ont marqué le $X X^{*}$ siècle : les exterminations d'Auschwitz, le lancement de la première bombe atomique sur Hiroshima, les décolonisations. "Chacun des grands récits d'émancipation, à quelque genre qu'il ait accordé l'hégémonie, a pour ainsi dire été invalidé dans son principe au cours des cinquante dernières années" ${ }^{2}$.

C'est a partir de la prise de conscience que les promesses de la modernité n'étaient pas réalisables que le discours postmoderne s'articule. Dans un premier temps, il annonce l'effondrement de l'adhésion populaire aux grands récits d'émancipation et ce, au profit d'une multiplication de microrécits. Ces derniers se différencient des premiers en ce qu'ils adoptent une approche narrative plutôt qu'un discours de vérité. Ils ne prétendent pas avoir une explication

1. Lyotard, J.-F. Le post-moderne expliquế aux enfants, Paris, éd. Gallilée, 1986, p. 78.

2. Ibid, p. 50 
universelle et incontournable; ils se contentent d'expliquer le particulier, le conjoncturel et le circonstanciel.

Si le discours de vérité échappe à ces microrécits, c'est tout simplement parce qu'il y a, selon Lipovetsky, une «déstandardisation et une démocratisation de la vérité" ${ }^{3}$. Ainsi, personne ne peut prétendre avoir l'exclusivité de la vérité absolue (c'est la chute de l'Avant-Garde). Chacun peut savoir ce qui lui convient ou pas. G. Scarpetta résume bien cette dimension de la postmodernité lorsqu'il affirme :

Le temps est révolu où certains pouvaient croire détenir la uposition juste" et les critères absolus, definitifs, permettant de brosser un tableau exact et complet de la situation culturelle l...la seule chose dont nous puissions être certains, c'est que toute une part de ce à quoi nous nous attachons finira par sombrer dans l'oubli, et que nous passons forcément à côté d'cuvres que l'avenir désenfouiral l... $\Gamma^{4}$.

Pour Gérard Raulet, cette dynamique postmoderne crée une importante "vacance normative" ${ }^{5}$ à l'intérieur de nos sociétés. C'est l'éclatement de "la" norme en d'innombrables normes qui dépendent presque exclusivement de "l'expressivité individuelle déréglée". La postmodernité doit alors être comprise comme l'époque du pluralisme et de l'éclectisme. Le pluralisme postmoderne se veut, selon

3. Lipovetsky, G., L'ère du vide, Paris, Gallimard, coll. «Folio/Essais», 1983.

4. Scarpetta, G., L'impureté, Paris, éd. Grasset, 1985, p. 21.

5. Raulet, G., «L'archipel, réflexion sur la démocratie postmoderne», dans Les Cahiers de philosophie, $n^{\circ} 6,1988, p .5$. 
l'expression de S. Campeau", "équilégitime" c'est-à-dire que, si tous les dialectes sont autorisés, aucun n'a la prééminence. Pour ce qui est de l'éclectisme, Charles Jenks ${ }^{7}$ affirme qu'il doit être interprété dans son sens étymologique de "Je choisis".

C'est à partir de cet éloge de l'éclectisme qu'il est permis de voir dans l'approche postmoderne une attitude démocratique qui s'appuie sur le sens de la responsabilité des individus, une responsabilité d'ordre pragmatique et non moral. D'ailleurs, Lyotard affirme que «la postmodernité serait plutôt empirico-critique ou pragmatiste»" ${ }^{8}$. De son côté, Lipovetsky ne cesse de soutenir que la démocratie est partie prenante de la postmodernité et déclare même qu'elle serait devenue la «seconde nature» de l'individu postmoderne. Si la démocratie nous semble aujourd'hui moins visible qu'elle ne l'était sous ses traits modernes (caractérisés par des revendications explicites), c'est tout simplement, toujours selon Lipovetsky, parce qu'elle est intrinsèquement liée au quotidien des individus. Pour ce penseur, l'époque des revendications démocratiques est révolue, et nous sommes maintenant dans "l'ère du vécu démocratique».

Etre un penseur postmoderne, c'est donc tenter de comprendre et d'interpréter les sociétés occidentales contemporaines dans leurs différentes composantes. C'est être l'ethnologue des sociétés capitalistes avancées.

\section{Critique de l'utopie et de la modernité}

La démarche intellectuelle de Havel est claire. Elle vise d'abord a faire reconnaître aux observateurs et aux intellectuels occidentaux que le mal post- totalitaire (régime communiste poststalinien) n'est pas un problème strictement

6. Campeau, S. * La raison postmoderne : sauver l'honneur du non?*, dans Philosopher, $n^{\circ} 8,1989$, p. 109-119.

7. Jencks, C., The Language of Post-modern Architecture, Londres, Academy Ed., 1987.

8. Lyotard, op.cit., p. 93 
extérieur à leur civilisation, mais qu'il s'agit d'abord et avant tout d'un mal occidental. Avec cet humour noir typiquement tchèque, le dramaturge-dissident s'est même permis d'avancer, dans une lettre adressée à l'intelligentsia française réunie à l'université Toulouse-le-Mirail, en 1984, que "le péril qui menace la culture occidentale vient moins des missiles SS-20, que de la culture occidentale elle-mêmen ${ }^{9}$.

Si Vaclav Havel reconnait, comme beaucoup d'autres commentateurs, que les bases historiques explicites du posttotalitarisme sont I'utopie socialiste du $\mathrm{XIX}^{\circ}$ siècle et l'obsession scientiste de Marx, il affirme que les racines implicites de ce mal sont enfouies dans l'inconscient occidental et qu'elles s'inscrivent directement dans le projet occidental lui-même.

Ce potentiel pervers enfoui dans les profondeurs de la culture occidentale réside, selon Havel, dans deux grands moments de la pensée occidentale : l'utopie platonicienne et le phantasme scientiste de la modernité qui en découle.

Afin d'approfondir cette prise de position qu'adopte Havel à l'égard de la philosophie occidentale, nous allons successivement examiner en détail sa critique de l'utopie et celle de la modernité.

\section{Critique de l'utopie}

Tout en reconnaissant que les idéaux sociaux et les rêves d'un monde meilleur sont des réactions tout à fait naturelles et même essentielles à la vie humaine, Havel recommande d'en user avec prudence. S'ils sont des éléments de base de la dignité humaine, donnant un sens à la vie et nous permettant d'affronter les lendemains incertains, ces idéaux peuvent très facilement devenir des utopies perverses et sanguinaires.

Continuant de jouer sur cette dualité positivité/danger, Havel affirme que le potentiel pernicieux du monde des idées ne réside pas dans la transcendance en soi, laquelle est souvent bénéfique aux individus. II tient plutôt de la mauvaise

9. Havel, V., a La politique et la conscience», dans Essais politiques, Paris, Calmann-Lévy, 1989, p. 242. 
habitude qu'a l'être humain de vouloir constamment matérialiser ses rêves parce qu'il les prend pour la réalité.

Ainsi, la métaphysique commence-t-elle a être dangereuse lorsque l'individu, trop fier de sa création intellectuelle pour la laisser dans le champ des idées, extorque l'idéal à la transcendance afin de le réaliser. C'est à partir de ce processus d'extorsion que l'idée devient utopie et qu'elle échappe peu à peu au contrôle de l'être humain.

Cette extorsion représente pour Havel «l'histoire tragique d'un court-circuit de la pensée". . La tragédie repose, selon lui, sur le désir légitime et constant qu'a l'être humain de vouloir soulager totalement sa vie des maux qui marquent son quotidien. Le drame commence quand on se convainc de pouvoir trouver remède a tous nos problèmes au moyen de notre gymnastique intellectuelle. Ainsi, l'utopie propose d'alléger le fardeau de nos responsabilités individuelles et civiques en trouvant une solution pour assurer une vie douillettement planifiée.

Le projet utopique, issu d'une bonne volonté, finit par menacer de travestir la nature réelle de l'être humain. "D'être d'interrogation" que nous sommes, le projet utopiste veut nous amener à un état artificiel «d'existence-réponse». Pour Havel, ce passage d'un état naturel à un état artificiel est le moment où la vie humaine commence à sombrer dans sa phase funeste.

Dans un texte qui s'adressait au Congrès d'Amsterdam pour la paix, en 1985, Havel exprime très clairement sa position critique à l'endroit de l'utopie. Il condamne la naïveté idéologique qui marque la pensée occidentale avec des arguments percutants, qui ont dô choquer plus d'un participant. Cette naïveté est telle, selon lui, que les Occidentaux sont prêts à prendre le premier train idéologique qui leur promet l'arrivée prochaine au paradis terrestre, cet état de paix perpétuelle (projet kantien) et universelle, cette harmonie globale. Ironisant sur ce thème, il dit :

De temps à autre apparaissent des individus qui ne supportent plus le chaos scandaleux de la vie et sa luxuriance mystérieuse. Ce sont des 
individus tragiquement tourmentés par l'horreur du néant et d'eux-mémes et par le besoin de se procurer le calme intérieur en établissant l'ordre (le "calme») dans le monde agité [...]. L'impatience désespérante de ces individus amène nécessairement à l'élaboration et à I'instauration de divers projets rationnels de bien-étre général, pour qu'on puisse tout comprendre, que le monde ait enfin une direction et que I'on en finisse définitivement avec tous les hasards de l'histoire. Mais a peine ont-ils commencé à agir dans ce sens [...], ils butent dejà contre les difficultés : beaucoup de leurs semblables veulent continuer de vivre a leur manière et, malgré sa perfection, le projet ne les intéresse pas $[. .$.$] . Bien sûr, le fanatique du$ projet abstrait, cet utopiste dans la pratique, ne peut tolérer une chose pareille [...]. C'est la raison pour laquelle il décide - évidemment dans l'intérêt du monde - d'imposer son projet par la force [...] il postule qu'il est juste de sacrifier quelques milliers de rebelles pour le bien de millions d'individus [...]. La conséquence nécessaire de tout cela est évidente : c'est le malheur pour tous ${ }^{10}$.

Après cette description de "l'haïssable" démarche de l'utopiste type, Havel ne s'est pas gêné pour rappeler les tristes souvenirs qu'ont laissés Marat, Robespierre, Lénine,

10. Havel, V., «Anatomie d'une résistance», dans Essais politiques, op. cit., p. 200-201. 
etc., ces grands utopistes qui ont fait couler tant de sang au nom de lendemains meilleurs.

La conclusion de sa critique de l'utopie est triple :

- Il faut d'abord critiquer et délaisser tous ceux qui prétendent être l'avant-garde éclairée, porteuse des lendemains meilleurs.

- Tout projet de société, même celui qui semble le plus ingénieux, doit être condamné dès l'instant où il nécessite la mort de «l'autre», voire d'un seul "autre». - II faut refuser toute volonté "d'organisation platonicienne de l'Europe future" ".

\section{Critique de la modernité}

On peut déjà sentir dans son analyse de l'utopie que l'essayiste Havel adopte une attitude très critique envers le rationalisme abusif, qu'il considère comme l'instrument par lequel l'Occident tente de matérialiser son vieux phantasme métaphysique.

C'est dans cette optique que de longs passages de ses essais sont consacrés à la critique de la modernité, la Mecque du rationalisme aveugle. Critique d'autant plus importante qu'elle permet, selon l'auteur, de comprendre que le phénomène post-totalitaire est la forme extrême de radicalisation de la pensée moderne.

La grisaille et le vide de la vie post-totalitaire ne sont-ils pas finalement l'image caricaturale de la vie moderne en général? Et ne sommes-nous pas en réalité... une espèce de mémento pour l'Occident, lui dévoilant sa tendance latente ${ }^{12}$

11. Ibid, p. 205

12. Havel, V., «Le pouvoir des sans pouvoirs», dans Essais politiques, op. cit., p. 86. 


\begin{abstract}
Le systeme post-totalitaire ne constitue que l'une des formes particulièrement sévère et n'en attestant que mieux la véritable origine - de cette incapacité générale de l'homme moderne à "être man̂tre de sa propre situation". La "gravitation" de ce systeme n'est qu'une version de la "gravitation" de la civilisation technique. L'échec humain qu'il reflète, qu'une variante de l'echec global de l'homme moderne ${ }^{13}$.
\end{abstract}

Si le post-totalitarisme découle de la logique moderne, il est très important que l'Occident prenne au sérieux ce dangereux glissement et cette radicalisation de l'âme moderne. Le post-totalitarisme est «le miroir convexe des conséquences nécessaires de ce rationalismen; il est le résultat de son abus extrême; il représente un signal d'alarme pour l'Occident.

Selon Havel, une chose pourrait quelque peu alléger la souffrance subie par les citoyens des pays de l'Est : leur malheureuse expérience doit servir de leçon à l'Occident face aux dangers que représente la frénésie moderne. L'Occident pourrait ainsi réaligner ses valeurs vers des objectifs plus humbles et plus humains. Malheureusement, se plaint Havel, les Occidentaux se complaisent tellement dans leur état d'être, et sont tellement peu ouverts à ce qu'ils considèrent comme leur "non-être», que l'on ne peut guère être très optimiste pour l'avenir.

Suivant la voie heideggerienne, Havel affirme que le probleme majeur à la source du vide post-totalitaire et du désarroi des sociétés occidentales est la crise générale des "sociétés techniciennes". Une crise caractérisée par l'impuissance globale que ressent l'être humain face à l'hégémonie sans cesse croissante de la technique sur

13. Ibid, p. 151. 
l'ensemble des activités humaines. Ainsi, l'homme sent qu'il ne maîtrise plus son environnement, sa société, ni lui-même. Le pire, c'est qu'il est incapable de trouver une solution pour sortir de son état d'asservissement. La puissance de la "culture technicienne" est telle qu'elle a réussi à convaincre l'être humain que le seul moyen de résoudre un problème est de lui appliquer une solution technique.

Devant ce constat, Havel prétend que le véritable problème n'est pas dans la technique en soi, dans la science en soi, ni même dans la modernisation, mais dans «l'orgueil de l'homme moderne", cause profonde de tous les maux de la modernité. Cette conviction moderne que «l'homme est maître absolu de la nature» a conduit aux portes de la tragédie. Dans un entretien avec Karel Hvizdala, Havel affirmait : *L'anthropocentrisme prétentieux de l'homme moderne, persuadé qu'il peut tout connaître et soumettre, me semble au fondement de la crise que nous traversons" ". C'est pourquoi une sérieuse remise en question de cet a priori moderne s'impose si l'on veut que l'humanité sorte du cul-de-sac dans lequel elle se trouve.

Gráce à la science, l'être humain a tenté de réaliser le vieux rêve de se faire Dieu. Porteur, par l'entremise de sa rigoureuse rationalité, de la vérité absolue, il s'est convaincu qu'il lui était dorénavant possible de matérialiser le paradis terrestre, cette société idéale depuis si longtemps attendue. Pour ce faire, l'homme moderne a dô s'attaquer à la démystification de la nature. Peu à peu, ce défi scientifique s'est transformé en campagne de dénigrement et de refus de la nature, considérée comme la caverne platonicienne où I'homme est condamné à vivre dans ses préjugés, donc dans une perpétuelle aliénation. Le nouveau mot d'ordre était donc : libérons-nous de la nature.

La toute-puissance de la rationalité moderne se trouvait désormais dans la connaissance objective, vérité universelle supérieure, suprasubjective, suprapersonnelle. Le second mot d'ordre était : émancipons-nous de l'humiliante subjectivité qui nous asphyxie. Ces attaques contre la subjectivité

14. Havel, V., Interrogatoire à distance, Paris, éd. de l'Aube, 1989 , p. 16. 
"maudite" s'adressaient aussi au Moi et à toutes les formes de responsabilité individuelle. Cette attaque dirigée contre le Moi était "une mise en parenthèse de l'homme concret", une dépersonnalisation globale des individus, ce qui nous mène en tant que tel au concept de «mort de l'homme» défini par Foucault. Ce travail de edésubjectivation", voire d'objectivation effrénée, a eu des répercussions très importantes sur l'avènement du post-totalitarisme, mais aussi de malencontreuses répercussions sur tout ce qui touche à l'immédiat, au vécu, au quotidien, univers où règnent l'amour, la haine et le respect, là où se vivent joies et douleurs.

En résumé, pour Havel, c'est à la vie en tant que telle que la modernité avait déclaré la guerre.

Bien entendu, le processus de rationalisation/ «désubjectivation" a eu des répercussions directes sur le pouvoir politique qui régente les sociétés modernes. Pour Havel, l'État et le pouvoir moderne doivent être compris comme "le moment où la Raison humaine se libère des hommes et de l'expérience" afin de s'incarner dans une instance objective et porteuse de la vérité absolue. Serait-ce le triomphe du schéma hégélien?

Ainsi, cette quête de l'instance objective suprême engendre un pouvoir anonyme que personne ne détient réellement et qui donne l'impression d'une gigantesque machine savante, d'un monstre scientifique inaccessible. II y a alors un culte de la "transparence des dirigeants" et une vénération du règne de l'abstraction lla bureaucratie, une idéologie, etc.).

Le pouvoir moderne est donc caractérisé par "l'expropriation des gouvernants et des gouvernés", ces derniers étant réduits à des fonctions anonymes et futiles, par exemple celles d' «électeurs, producteurs, consommateurs, malades, etc.", tandis que les premiers sont confinés à des rôles d'accessoires : "managers, apparatchiks, bureaucrates, etc.".

Sur le plan de son action, le pouvoir moderne se limite à une simple "technique de domination et de manipulation» reposant sur une objectivité froide et impartiale. Ce n'est que l'aspect fonctionnel et utilitaire d'une chose qui compte pour ce pouvoir. II se fait donc amoral, car le bien et le mal 
perdent tout leur sens à l'intérieur des critères fonctionnalistes.

Si le post-totalitarisme est le produit extrême de cette perversité moderne qu'est le pouvoir impersonnel, les régimes parlementaires occidentaux ne doivent pas croire qu'ils en sont à l'abri.

Comme Heidegger, Havel parle de la crise majeure qui frappe les démocraties occidentales. Certes, la crise de ces démocraties paraît moins grave que celle des pays de l'Est, car il y a en Occident une plus grande protection des droits et libertés individuels. Toutefois, cette protection, Havel la juge superficielle, car les systèmes occidentaux restent fondés sur la méfiance à l'endroit de la subjectivité, ce qui conduit directement à la confiscation croissante des responsabilités civiques individuelles.

Pour Havel, le règne de l'irresponsabilité, qui caractérise les démocraties parlementaires, prive les citoyens occidentaux d'une réelle liberté ou, dans une moindre mesure, limite grandement cette liberté. À propos de ces démocraties occidentales, Havel écrit :

Tout cet ensemble statique de partis de masses sclérosés et agissant politiquement de manière tellement intéressée, ces partis dominés par des appareils professionnels qui dechargent le citoyen de toutes responsabilités concrètes et individuelles. [...] // [le citoyen] est victime de gravitation, il est incapable de préserver son identité, de se protéger de son aliénation et de dépasser le cadre de ses soucis de survie individuelle, pour devenir membre responsable de la "polis" et fier de l'être, participant à la construction de son destin ${ }^{15}$.

15. Ibid., p. 151-152. 
Malgré ces critiques acerbes à l'endroit des régimes parlementaires, Havel reconnaît que les démocraties parlementaires peuvent être considérées comme des systèmes de transition viables, grâce notamment à la pluralité du multipartisme. Ces systèmes de transition devraient nous mener vers des modeles politiques plus complets, qui reposeraient globalement sur la responsabilité civique de tous les membres de la communauté.

En ce sens, il reproche aux régimes parlementaires leur prétention narcissique à des régimes politiques qui constituent des fins en soi. Selon Havel, c'est une erreur de croire que la dignité des individus se conquiert ou se défend uniquement de manière "constitutionnelle».

Redéfinition du concept de liberté

Dans sa critique des démocraties parlementaires, Havel laisse entrevoir l'idée qu'il se fait de la liberté, c'est-à-dire une composante essentielle de la vie concrete, aux antipodes du principe de mort qui se terre sous la notion d'ordre. La vie humaine doit être reconnue comme mouvement et mystère, c'est-à-dire comme une incarnation de l'insaisissable, comme l'essence même du concept de liberté humaine.

Pour l'essayiste, le monde humain est un lieu immense où s'assemblent d'innombrables sujets différents, où intervient le jeu «imprévisible des interactions de pluralité de sujets relativement indépendants". Au lieu de nier cette réalité marquée par la différence, comme le fait l'universalisme moderne, il est au contraire essentiel de reconnaître la richesse de la diversité. Le pluralisme, qui découle de ces différences, est une valeur essentielle à l'organisation des sociétés humaines. Une telle prise de position ne peut que réconforter les tenants de la thèse postmoderne qui repose sur la primauté de ce pluralisme social.

Le respect de la pluralité est la base d'un sain épanouissement de la liberté individuelle. Havel affirme que, «là où il n'y a pas plusieurs singularités, il n'y en a 
aucune» ${ }^{10}$. II pense également que la liberté individuelles implique la singularité.

Ce droit à la différence, comprise comme une valeur sociale, nécessite également la réintégration de la valeur d'égalité au sens d'égalité des différences et non à celui de nivellement comme on a trop souvent eu tendance a le penser. En effet, si l'on veut réellement respecter les diversités, il faut avant tout reconnaître que les différentes spécificités sont égales. "ll n'y aura pas de liberté sans égalité et pas d'égalité sans liberté" dira Havel.

Si la liberté et l'égalité sont dorénavant liées dans un pluralisme social, il est essentiel, insiste Havel, de concevoir la liberté et les droits individuels comme indivisibles. Tous doivent comprendre "qu'une atteinte a la liberté d'un seul porte atteinte à la liberté de tous" et toutes les libertés doivent être considérées comme fondamentales. En négliger une seule serait une menace pour toutes les autres. Même si certaines formes de liberté peuvent nous paraitre marginales (il cite l'exemple de la liberté de jouer de la musique rock), l'on ne peut pas les laisser être brimées en raison de leur moindre importance, sinon toutes les autres formes de liberté s'en ressentiront.

\section{La liberté doit être Une, ou elle ne sera pas!}

Pour que la liberté réelle puisse s'implanter, une double exigence morale et politique s'impose aux individus.

Á la base, la liberté ne peut s'épanouir qu'au milieu de la "vie dans la vérité». Il est essentiel que la réalité concrète soit constamment manifeste. Aucun citoyen ne doit fermer les yeux et se taire devant un élément ou un fait de la vie quotidienne.

Cette position morale ne doit cependant pas être un vœu pieux. Pour assurer l'épanouissement véritable de la vie dans la vérité, il est nécessaire de mettre en place un processus qui permette et protège l' «accès a l'information et a la confrontation des idées". C'est par la libre circulation des

16. Havel, V., *Histoire et totalitarisme*, dans Essais politiques, op. cit., p. 175. 
idées, de l'information, des connaissances, des valeurs, etc., que la vie dans la vérité peut concrètement exister et non dans une pseudo-idée éclairée préconçue.

Même si toutes les libertés sont égales, Havel reconnaît que la liberté d'expression en est "la plus haute forme", car elle permet aux différences de s'exprimer librement, et à la communauté de vivre dans la vérité. La liberté d'expression préserve la pluralité contre l'homogénéisation.

Autre critère qui assure le sain épanouissement de la liberté : la responsabilité individuelle. Pour Havel, sans l'essor de la responsabilisation, toute liberté est illusoire. Dans son

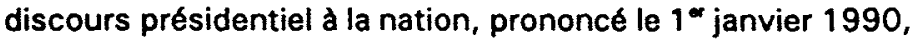
Vaclav Havel a insisté sur ce point :

Détrompons-nous : le meilleur
gouvernement, le meilleur parlement
et le meilleur président ne peuvent
pas, à eux seuls, faire grand chose.
Et ce serait injuste d'attendre la
solution d'eux seulement. La liberté
et la démocratie, cela signifie la
participation et la responsabilité de
tous ${ }^{17}$.

Cette responsabilité concerne d'abord la défense de nos droits et libertés contre quiconque voudrait les menacer. L'individu doit se rendre compte de ce que le renoncement a ses responsabilités publiques au profit d'autorités politiques conduit à l'effritement et à la négation de ses libertés.

Comme les droits et libertés sont indivisibles, le principe de responsabilité oblige l'individu à ne plus se replier sur luimême et à s'intéresser aux choses qui débordent sa sphère privée. Loin de tomber dans l'altruisme, l'individu doit comprendre que la défense de sa sphère privée se fait également, voire prioritairement, sur le plan public. C'est ainsi

17. Havel, V., «Vœux pour la Tchécoslovaquie*, dans $L$ 'amour et la vérité doivent triompher de la haine et du mensonge, Paris, éd. de l'Aube, 1990, p. 27. 
qu'il se réapproprie le statut de "sujet actif de l'histoire" et le privilège d'être citoyen.

Ce retour du sentiment civique est primordial, dans la mesure où il engendre une reprise en main des destinées publiques par l'ensemble des citoyens. C'est la réapparition de la conscience de coresponsabilité des affaires publiques. Lors de sa première grande réapparition publique durant la «révolution de velours», Vaclav Havel déclara :
Je m'adresse à vous en tant que chacun d'entre vous, c'est-à-dire en tant que citoyen de ce pays qui ressent la coresponsabilité de son destin futur ${ }^{18}$.

C'est grâce à cette conscience civique que la "chose publique» redeviendra réellement l'affaire de tous, car le civisme doit réapparaître sur le plan de la collectivité et non sur le plan personnel :
L'aspiration de taire valoir notre propre droit à la citoyenneté implique essentiellement le droit d'autrui à cette même qualité. Qu'un seul homme soit privé de ce droit, cela revient à le dénier à tous. II s'ensuit que les citoyens sont - en tant que citoyens - egaux par principe devant la loi, la société et le pouvoir ${ }^{19}$.

L'intérêt du retour à la responsabilité civique réside dans sa triple incidence. Tout d'abord, la société cesse peu à peu d'être atomisée et ce, grâce au renforcement des liens sociaux engendré par la remise en place des valeurs civiques.

18. Havel, V., «Pourquoi je suis candidat», dans L'amour et la vérité doivent triompher de la haine et du mensonge, op. cit., p. 23.

19. Havel, V., «Le sens de la Charte 77», dans Essais politiques, op. cit., p. 55. 
Ensuite, l'individu "retrouve son intégrité, sa dignité et le respect de soi-même» grâce au sentiment de fierté qu'il ressent en redevenant un sujet actif et en reprenant son droit de parole dans la direction du devenir social. Enfin, cette coresponsabilité redonne à l'organisation sociale son véritable visage démocratique qu'Havel nommera plus loin : postdémocratie.

La réflexion que consacre Havel au retour du civisme est des plus intéressantes en tant que projet intellectuel de renouvellement de la démocratie. Nous sommes cependant obligés de reconnaître qu'elle est aussi une rechute dans I'utopie. En effet, si Havel s'était contenté de promouvoir un retour en force d'un certain niveau de responsabilisation des individus, sa démarche aurait été tout à fait louable. Malheureusement, en préconisant la toute-puissance du civisme, Havel sombre dans un fétichisme plutôt dangereux.

Deux brèves citations expriment tout de même l'importance que l'ex-président tchèque accorde au principe de responsabilité civile :

Seuls des citoyens libres, conscients de leurs droits, pourront concevoir, formuler et defendre de nouveaux programmes politiques [...]. Sans citoyen, il n'y a pas de politique ${ }^{20}$.

Sans citoyens dignes, libres et émancipés, il n'y a pas de peuples libres et indépendants. Sans paix intérieure, c'est-à-dire entre les citovens eux-mémes et entre les citoyens et l'État, il n'y a pas de garantie de paix extérieure ${ }^{21}$.

20. Ibid., p. 51.

21. Havel, V., *Anatomie d'une réticence», dans Essais politiques, op. cit., p. 211. 
Vers la postdémocratie

Au long de sa réflexion sur la primauté de la responsabilité civique, Havel réitère la nécessité d'une révision en profondeur du politique. Cette métamorphose sera tellement importante qu' on ne pourra plus parler de politique proprement dite, mais plutôt de upolitique antipolitique". II lui semble indispensable de mettre en place une nouvelle organisation où les sujets individuels pourront être maitres de leurs actions et de leurs décisions. Le politique, chambardé par le sentiment généralisé de coresponsabilité, devrait adopter une structure horizontale et non la traditionnelle hiérarchie verticale des systèmes actuels. Ce projet de structure horizontale $n$ 'a cependant rien de nouveau et ressemble étrangement au projet des socialistes utopistes. Un tel rapprochement n'est pas étonnant puisque, dans Interrogatoire à distance, Havel explique qu'il a longtemps été un partisan convaincu de ce type de socialisme.

La politique horizontale, ou politique d'en bas, doit prendre racine dans l'homme concret et se donner pour tåche de travailler a l'amélioration substantielle de la vie quotidienne, c'est-à-dire le "ici maintenant". Ainsi, l'être humain cessera d'être au service de l'organisation sociale, et c'est dorénavant celle-ci qui sera assujettie aux individus. Pour Havel, ce renversement est nécessaire, car la bonification concrète de la vie humaine ne peut découler que d'une volonté commune des individus.

C'est une erreur de croire que le bonheur et la vie meilleure peuvent être le simple fruit des lois, d'une constitution ou même d'une organisation : «La solution pour une vie humaine digne, riche et heureuse ne se trouve pas dans une constitution ou dans un code pénals ${ }^{22}$. Les lois, la constitution et l'organisation sociale ne sont pas des fins en soi, et les considérer ainsi peut mener à des atrocités, hélas connues. Elles ne sont que de simples moyens de «protéger la vie humaine contre le pire», et ne doivent être que le reflet de la vie quotidienne réelle. Elles doivent se limiter à un rôle

22. Havel, V., «e pouvoir des sans-pouvoir*, op. cit., p. 134. 
moral lié à la vie; leur indépendance constitue une entrave à la liberté et à la vie.

La révision du politique n'est pas une mince tâche, car il ne faut pas croire que cet imposant processus de transformation sociale puisse se faire immédiatement et sans difficulté. Au contraire, pour aboutir à un tel type d'organisation sociale, où l'homme serait à la base même de toute la structure, une importante "révolution existentielle» s'impose chez les membres de la communauté :

La perspective de la "révolution
existentielle" représente surtout -
pour ce qui est des conséquences -
une perspective de reconstitution
morale de la société, autrement dit
un renouvellement radical de la
relation authentique de l'individu àce
que j'ai appelé "l'ordre humain"-et
qui ne peut étre supplée par aucun
ordre politique: une nouvelle
expérience de l'être, un nouvel
enracinement dans l'univers, une
"responsabilité supérieure" a
nouveau saisie, une relation intérieure
a autrui et a la communauté

La "révolution existentielle" ou "renaissance spirituelle " ${ }^{24}$ représente l'étape qui, selon $\mathrm{Havel}$, et ce, dans la pure tradition phénoménologique héritée de Patochka lle hursellien), permet à l'individu de redonner un sens à son existence. Celui-ci prend ainsi conscience de ce qu'il peut redevenir un être actif et essentiel pour le sain épanouissement de sa communauté et donc de ses semblables; il reprend confiance en lui et découvre un nouveau lien émotif avec sa communauté.

23. Ibid., p. 153.

24. Havel, V., Interrogatoire d distance, op. cit., p. 17. 
Pour contrer les critiques qui pourraient l'accuser de retomber dans une approche religieuse ou messianique, Havel précise que cette révolution existentielle ne va pas tomber du ciel ou être apportée par un nouveau Messie. C'est nous tous qui, ici et maintenant, pouvons et devons «faire quelque chose». Personne ne le fera a notre place et nous ne devons compter sur aucune aide ${ }^{25}$. Pour avoir une répercussion directe et concrète sur la vie en communauté, la révolution existentielle doit pouvoir s'appuyer sur un renouvellement de la vocation de la politique. Il s'agit de transformer la tribune politique en un lieu intermédiaire entre la sphère transcendante, où l'individu donne un sens à sa vie, et la vie pratique quotidienne. Ainsi, la politique se voit attribuer un rôle de morale appliquée, dont la fonction première est de redonner un sens à la richesse de la vie en communauté humaine.

Un premier moyen d'y parvenir est de mettre la morale au-dessus de la politique traditionnelle et la responsabilité individuelle au-dessus de l'utilitarisme social, proposition qui rappelle la morale politique kantienne. II s'agit donc de réhabiliter des valeurs sociales et morales telles que la confiance, la vérité, l'amour, la solidarité, l'ouverture. C'est à partir de ces valeurs morales que la politique antipolitique doit se mettre en place. Ce n'est donc plus la politique qui dicte les valeurs sociales, mais plutôt la morale qui dicte au politique sa ligne de conduite :

La politique telle que je la comprends est une des manières de chercher et d'acquérir un sens dans la vie; une des manières de protéger et de servir ce sens; c'est la politique comme morale agissante, comme service de la vérité, comme souci du prochain, souci essentiellement humain, réglé par des critères humains ${ }^{28}$.

25. Ibid.

26. Havel, V., "La politique et la conscience», op. cit., p. 245. 
L'orientation morale de l'écrivain s'inscrit dans la plus pure tradition philosophique et culturelle tcheque. Tradition qui, depuis Jan Hus (le grand réformateur politique du Moyen Âge), vise à unir philosophie et politique dans un projet de morale appliquée, afin d'assurer l'épanouissement de la nation tchèque ${ }^{27}$.

Si Havel est conscient du fait que sa vision politique est, dans l'immédiat, difficilement réalisable à cause du niveau élevé de corruption au sein de tout régime politique, il ne la considère pas comme utopique, parce qu'elle s' appuie sur des valeurs très simples et non sur un grand projet intellectuel ou métaphysique. Certes, Havel a raison : la confiance, la vérité, l'amour sont des valeurs très simples, beaucoup trop simples pour être à la base du renouveau de nos sociétés qui ne cessent de se complexifier. Ces valeurs sont d'une telle simplicité et d'une telle évidence qu'elles ont tout pour être à la base d'un nouveau fléau utopique. La Révolution française n'a-t-elle pas sombré dans le carnage pour faire triompher la vertu? Au nom de la vérité, de la confiance, de l'amour, la révolution existentielle ne risque-t-elle pas de sombrer dans un piège de cette nature?

Pour qu'un tel projet puisse se réaliser, il faudrait, pense Havel, réviser toute l'organisation sociale et les structures politiques qui la composent. D'où son projet de postdémocratie.

Bien qu'il affirme avoir horreur de spéculer sur le futur, Havel trace un schéma des structures politico-sociales idéales pour l'avenir des communautés.

Pour s'assurer que la pluralité des vérités, des volontés et des décisions puisse s'exprimer librement, le système politique doit être caractérisé par un pluralisme politique et un important équilibre des pouvoirs. Les structures politico-sociales doivent découler d'une ambition (d'une Idée au sens kantien) d'auto-organisation sociale; elles doivent être ouvertes, variées, de petites dimensions et le moins

27. Pour plus de détails sur ces influences philosophiques et culturelles tchèques, voir Havel, $V$., Interrogatoire d distance, op. cit. , ou Boisvert, $Y .$, a Vaclav Havel : président, dramaturge et philosophe», dans L'analyste, hiver 1991-1992, n’36, p. 25-28. 
régulatrices possible. Elles doivent représenter de façon réelle les préoccupations immédiates et concrètes qui sont au cœur de la communauté. En ce sens, elles doivent être conjoncturelles et disparaître graduellement au profit de nouvelles structures adaptées aux nouveaux problèmes affectant les membres de la communauté.

Le but ultime de ces structures souples et maniables est de court-circuiter toute éventualité de concentration et d'accumulation de pouvoir. Le jeu politique est donc marqué par un changement régulier de personnel et de groupes influents. La dynamique sociale sera ainsi continuellement en mouvement; I'autodirection et la coresponsabilité seront les uniques liens politiques de ces communautés.

Ce modèle, qui s'appuie sur des structures sociales conjoncturelles, se rapproche beaucoup de la théorie des nouveaux mouvements sociaux (Melucci) et d'autres théories contemporaines qui s'intéressent aux nouveaux phénomènes sociaux comme le postmodernisme.

Sur le plan économique, il n'est pas étonnant de voir Havel ressusciter ce qu'il appelle ule vieux rêve socialiste" de l'autogestion basée sur une participation réelle des travailleurs aux décisions économiques et sur une véritable coresponsabilité face au produit de leur travail.

On remarque donc que l'idéal postdémocratique élaboré par Havel comporte une forte résonnance phénoménologique. Tout repose sur la primauté de la signification profonde que les individus accorderont aux activités qu'ils entreprendront. Cette primauté du sens personnalise l'action et la rend humaine, et, de cette manière, il devient possible de réhumaniser ce monde impersonnalisé par la pensée moderne.

\section{Conclusion}

Au terme de cette brève synthèse de la philosophie politique de Vaclav Havel, la première question qui nous vient à l'esprit est celle-ci : devons-nous conclure que cet écrivain prône la liquidation pure et simple de la modernité? Si oui, pouvons-nous parler de pensée postmoderne?

S'il est vrai qu'il n'a jamais explicitement parlé d'élimination globale de la modernité, il faut cependant 
reconnaître que ses critiques cinglantes à l'égard de celle-ci laissent croire que l'ex-président de la Tchécoslovaquie peut sembler favorable à un dépassement de la modernité. En s'appuyant sur certains de ses propos (par exemple : «On n'a jamais encore réussi à éliminer un mal en éliminant ses symptômes. C'est la cause qu'il faut supprimer»), des commentateurs plus radicaux pourraient facilement démontrer que Havel ne réclame rien de moins que la liquidation pure et simple de la modernite. Antimoderne? Postmoderne? La question reste nébuleuse.

Cependant, quand on exige un rejet sans condition de la prémisse "l'homme maître de la nature" , que l'on réinsère le mystère pour contrer le dogmatisme de la vérité absolue, et que l'on insiste sur la subjectivité pour mettre un frein au fléau de l'objectivisme dépersonnalisant, peut-on encore être un penseur moderne? La question est ouverte!

Peu importe notre réponse à la question précédente, on ne peut nier que les écrits philosophico-politiques de l'exprésident apportent de la substance théorique au $x$ adversaires de la modernité, voire de la postmodernité. Ses critiques des projets platoniciens et cartésiens sont, sur plusieurs plans, similaires à celles du postmodernisme, tout comme son rejet du dogmatisme et de la métaphysique. Le parallélisme de sa pensée avec celle des tenants de la postmodernité est encore plus visible dans sa profession de foi différentialiste et pluraliste. Sa problématique de "l'égalité des différences» s'inscrit également dans le moule de la postmodernité.

Son parti pris pour la responsabilisation des individus et pour une démocratie plus directe rejoint des a priori postmodernes. Havel s'éloigne cependant du postmodernisme lorsqu'il insiste sur l'importance du spirituel qui doit sous-tendre ce processus. En aucun cas, la postmodernité ne pourrait admettre la nécessité d'une révolution spirituelle pour apporter les changements voulus. Pour les postmodernes, la responsabilisation et la démocratisation sont des résultats naturels qui $n$ 'ont nullement été provoqués.

Le "totémisme" de Havel à l'endroit de la morale n'est pas postmoderne. La pensée postmoderne ne pourrait tolérer de se laisser enfermer dans le carcan de la morale dont la saveur transcendante et l'esprit normatif sont beaucoup trop 
prononcés. On peut affirmer que le discours postmoderne s'opposerait vigoureusement au principe havélien de la "morale au-dessus de la politique». Le schéma politique postmoderne montre plutôt la politique comme une instance strictement pragmatique, exempte de toute morale. Avec une société caractérisée par une "vacance normative", la politique postmoderne ne peut être qu'amorale. Elle ne peut se permettre d'imposer quelque morale que ce soit à l'ensemble de la société. Dans la postmodernité, la moralité est une affaire privée.

Si Havel prend ses distances à l'égard de la métaphysique, il demeure, par la primauté qu'il accorde à la morale, tributaire d'une pensée de la transcendance. Plusieurs de ses lecteurs pourraient même $y$ voir un relent de religiosité. Nous croyons qu'il est plus juste de parler d'un retour à une forme de spiritualité. Mais, assurément, la pensée de Vaclav Havel se situe aux antipodes du nihilisme ou du fatalisme. Pour lui, la croyance demeure l'un des attributs fondamentaux du genre humain, et il lui est nécessaire de croire que la vie a un sens. Cet aspect spiritualiste et phénoménologique de la pensée de Havel entre également en conflit avec l'incrédulité des postmodernes pour qui toute croyance n'est que partielle. La croyance postmoderne se réduit au rêve et, comme le dit G. Vattimo en paraphrasant Nietzsche, "certes il faut continuer de rêver, mais il faut demeurer conscient que l'on rêve!» La postmodernité est également aux antipodes de la phénoménologie, car, pour elle, toute chose n'a de sens que celui que l'on lui donne, et l'essence des choses est réduite à n'être que le produit de l'imagination personnelle.

En réinsérant la spiritualité dans l'organisation sociale et en accordant la primauté à la morale, Havel se rapproche beaucoup plus de la pensée communautarienne de $A$. Macintyre (After Virtue). Lorsqu'il base son projet de politique antipolitique sur des valeurs sociales et morales telles que l'amour, la solidarité, l'ouverture, il se situe aussi dans la logique communautarienne, grandement influencée par la pensée aristotélicienne et teintée d'un antimodernisme beaucoup plus prononcé que celui du postmodernisme. Par leur acharnement a faire de la vertu la base même de la 
communauté, les tenants de la pensée communautarienne se font constamment taxer d'utopisme naïf.

Après sa critique acerbe de l'utopie, Vaclav Havel aurait dû faire attention pour ne pas tomber dans le piège communautarien.

$\mathrm{Si}$, au début de son mandat présidentiel, Vaclav Havel paraissait confiant de pouvoir mettre en place certains jalons de sa politique antipolitique, il a semblé depuis quelque temps de plus en plus déçu par la pratique politique et désabusé face au problème constitutionnel de son pays.

Les forces nationalistes slovaques ont finalement eu raison de la morale politique de $\mathrm{Havel}$. Les Slovaques ont en effet préféré l'autonomie politique à la promesse d'harmonie et d'égalité des différences que leur proposait l'ancien président. 